\title{
Global Identification of Drive Gains Parameters of Robots Using a Known Payload
}

\author{
Maxime Gautier and Sébastien Briot
}

\begin{abstract}
Off-line robot dynamic identification methods are based on the use of the Inverse Dynamic Identification Model (IDIM), which calculates the joint forces/torques that are linear in relation to the dynamic parameters, and on the use of linear least squares technique to calculate the parameters (IDIM-LS technique). The joint forces/torques are calculated as the product of the known control signal (the current reference) by the joint drive gains. Then it is essential to get accurate values of joint drive gains to get accurate identification of inertial parameters. In the previous works, it was proposed to identify each gain separately. This does not allow taking into account the dynamic coupling between the robot axes. In this paper the global joint drive gains parameters of all joints are calculated simultaneously. The method is based on the total least squares solution of an over-determined linear system obtained with the inverse dynamic model calculated with available current reference and position sampled data while the robot is tracking one reference trajectory without load on the robot and one trajectory with a known payload fixed on the robot. The method is experimentally validated on an industrial Stäubli TX-40 robot.
\end{abstract}

\section{INTRODUCTION}

S EVERAL schemes have been proposed in the literature to identify the dynamic parameters of robots [1]-[7]. Most of the dynamic identification methods have the following common features:

- the use of an Inverse Dynamic Identification Model (IDIM) which calculates the joint force/torque linear in relation to the dynamic parameters,

- the construction of an over-determined linear system of equations obtained by sampling IDIM while the robot is tracking some trajectories in closed-loop control,

- the estimation of the parameter values using least squares techniques (LS). This procedure is called the IDIM-LS technique.

The experimental works have been carried out either on prototypes in laboratories or on industrial robots and have shown the benefits in terms of accuracy in many cases. Good results can be obtained provided two main conditions are satisfied:

- a well-tuned derivative band-pass filtering of joint position is used to calculate the joint velocities and

Manuscript received September 15, 2011.

M. Gautier is with the University of Nantes and the Institut de Recherche en Communications et Cybernétique de Nantes (IRCCyN) 44321 Nantes France (phone: +33240376960; fax: +33240376930; e-mail: Maxime.Gautier@irccyn.ec-nantes.fr).

S. Briot is with the CNRS and IRCCyN, 44321 Nantes France (e-mail: Sebastien.Briot@irccyn.ec-nantes.fr). accelerations,

- the accurate values of joint drive gains $g_{\tau}$ are known to calculate the joint force/torque as the product of the known control signal calculated by the numerical controller of the robot (the current references) by the joint drive gains [8].

This needs to calibrate the drive train constituted by a current source amplifier with gain $G_{i}$ which supplies a permanent magnet DC or a brushless motor with torque constant $K_{t}$ coupled to the link through direct or gear train with gear ratio $N$.

Because of large values of the gear ratio for industrial robots, $(N>50)$, joint drive gain, $g_{\tau}=N G_{i} K_{t}$, is very sensitive to errors in $G_{i}$ and $K_{t}$ which must be accurately measured from special, time consuming, heavy tests, on the drive chain [8][9].

Several papers on the topic of the joint drive gains identification have been published in the past [8]-[11], but all of them propose to identify each gain separately. This does not allow taking into account the dynamic coupling between the robot axes.

In this paper it is proposed a new method for the global identification of the joint drive gains, using current reference and position sampled data while the robot is tracking one reference trajectory without load fixed on the robot and one trajectory with a known payload fixed on the robot whose inertial parameters are measured. Contrary to the previous works, all drive gains are calculated in the same solving loop by the total LS solution of an over-determined system in order to take into account the coupling between the robot axes.

The method is experimentally validated on an industrial Stäubli TX-40 robot.

The paper is organized as follows: section 2 recalls the dynamic modelling and identification procedures. Section 3 deals with the new modelling and identification method for the robot drive gains parameters. Section 4 presents the experimental validations. Finally, section 5 gives the conclusion.

\section{USUAL INVERSE DYNAMIC MODELS AND IDENTIFICATION}

\section{A. Inverse Dynamic Identification Model (IDIM)}

It is known that the dynamic model of any manipulator can be linearly written in term of a $(n \times 1)$ vector of standard 
parameters $\chi_{s t}$ [2], [4], [5], [11]. The modified Denavit and Hartenberg notation allows obtaining a dynamic model that is linear in relation to a set of standard dynamic parameters, $\chi_{s t}$ :

$\tau_{i d m}\left(q, \dot{q}, \ddot{q}, \chi_{s t}\right)=\Phi_{s t}(q, \dot{q}, \ddot{q}) \chi_{s t}$

where:

$\tau_{i d m}$ is the $(n \times 1)$ vector of the input efforts

$\Phi_{s t}$ is the $\left(n \times n_{s t}\right)$ jacobian matrix of $\tau_{i d m}$, with respect to the $\left(n_{s t} \times 1\right)$ vector $\chi_{s t}$ of the standard parameters given by $\chi_{s t}=\left[\begin{array}{llll}\chi_{s t}^{1 T} & \chi_{s t}^{2 T} & \ldots & \chi_{s t}^{n T}\end{array}\right]^{T}$

$q, \dot{q}, \ddot{q}$ are the vectors of the joint positions, velocities and accelerations, respectively.

For rigid robots, there are 14 standard parameters by link and joint. For the joint and link $j$, these parameters can be regrouped into the $(14 \times 1)$ vector $\chi_{s t}^{j}[5]$ :

$\chi_{s t}^{j}=\left[X X_{j} X Y_{j} X Z_{j} Y_{j} Y Z_{j} Z_{j} M X_{j} M_{j} M_{j} M_{j} I a_{j} F v_{j} F c_{j} \tau_{d f f_{j}}\right]^{T}$

where:

$X X_{j}, X Y_{j}, X Z_{j}, Y Y_{j}, Y Z_{j}, Z Z_{j}$ are the 6 components of the inertia matrix of link $j$ at the origin of frame $j$.

$M X_{j}, M Y_{j}, M Z_{j}$ are the 3 components of the first moment of link $j, M_{j}$ is the mass of link $j, I a_{j}$ is a total inertia moment for rotor and gears of actuator $j$.

$F v_{j}, \quad F c_{j}$ are the visquous and Coulomb friction coefficients of the transmission chain, respectively,

$\tau_{\text {off }}=\tau_{o f f F S_{j}}+\tau_{o f f \tau_{j}}$ is an offset parameter which regroups the amplifier offset $\tau_{\text {off } \tau_{j}}$ and the asymmetrical Coulomb friction coefficient $\tau_{o f f F S_{j}}$.

The identifiable parameters are the base parameters which are the minimum number of dynamic parameters from which the dynamic model can be calculated. They are obtained from the standard inertial parameters by regrouping some of them by means of linear relations [15], which can be determined for the serial robots using simple closed-form rules [3], [5], or by numerical method based on the QR decomposition [14].

The minimal dynamic model can be written using the $n_{b}$ base dynamic parameters $\chi$ as follows:

$\tau_{i d m}=\Phi(q, \dot{q}, \ddot{q}) \chi$

where $\Phi$ is obtained from $\Phi_{s t}$ by eliminating the columns corresponding to the non identifiable parameters.

Because of perturbations due to noise measurement and modelling errors, the actual force/torque $\tau$ differs from $\tau_{i d m}$ by an error, $e$, such that:

$\tau=\tau_{i d m}+e=\Phi(q, \dot{q}, \ddot{q}) \chi+e$

where: $\tau=v_{\tau} g_{\tau}=\left[\begin{array}{ccc}v_{\tau}^{1} & 0 & 0 \\ 0 & \ddots & 0 \\ 0 & 0 & v_{\tau}^{n}\end{array}\right]\left[\begin{array}{c}g_{\tau}^{1} \\ \vdots \\ g_{\tau}^{n}\end{array}\right]$

$v_{\tau}$ is the $(n \times n)$ matrix of the actual current references of the current amplifiers ( $v_{\tau}^{j}$ corresponds to actuator $j$ ) and $g_{\tau}$ is the $(n \times 1)$ vector of the joint drive gains $\left(g_{\tau}^{j}\right.$ corresponds to actuator $j$ ). Equation (4) represents the IDIM.

\section{B. Least Squares Identification of the Dynamic Parameters} (IDIM-LS)

The off-line identification of the base dynamic parameters $\chi$ is considered, given measured or estimated off-line data for $\tau$ and $(q, \dot{q}, \ddot{q})$, collected while the robot is tracking some planned trajectories. The model (4) is sampled and low pass filtered in order to get an over-determined linear system of $(n \times r)$ equations and $n_{b}$ unknowns:

$Y(\tau)=W(\hat{q}, \hat{\dot{q}}, \hat{\ddot{q}}) \chi+\rho$

where

$(\hat{q}, \hat{\dot{q}}, \hat{q})$ are an estimation of $(q, \dot{q}, \ddot{q})$, obtained by band-pass filtering and sampling the measure of $q$ [16].

$\rho$ is the $(r \times 1)$ vector of errors,

$W(\hat{q}, \hat{\dot{q}}, \hat{q})$ is the $\left(r \times n_{b}\right)$ observation matrix.

Using the base parameters and tracking "exciting" reference trajectories, a well conditioned matrix $W$ is obtained. The LS solution $\hat{\chi}$ of (6) is given by:

$\hat{\chi}=\left(\left(W^{T} W\right)^{-1} W^{T}\right) Y=W^{+} Y$

It is computed using the $\mathrm{QR}$ factorization of $W$.

Standard deviations $\sigma_{\hat{\chi}_{i}}$, are estimated assuming that $W$ is a deterministic matrix and $\rho$, is a zero-mean additive independent Gaussian noise, with a covariance matrix $C_{\rho \rho}$, such that:

$C_{\rho \rho}=E\left(\rho \rho^{\mathrm{T}}\right)=\sigma_{\rho}^{2} I_{r}$

$E$ is the expectation operator and $I_{r}$, the $(r \times r)$ identity matrix. An unbiased estimation of the standard deviation $\sigma_{\rho}$ is:

$\hat{\sigma}_{\rho}^{2}=\|Y-W \hat{\chi}\|^{2} /(r-b)$

The covariance matrix of the estimation error is given by: $C_{\hat{\chi} \hat{\chi}}=E\left[(\chi-\hat{\chi})(\chi-\hat{\chi})^{\mathrm{T}}\right]=\hat{\sigma}_{\rho}^{2}\left(W^{\mathrm{T}} W\right)^{-1}$.

$\sigma_{\hat{\chi}_{i}}^{2}=C_{\hat{\chi} \hat{x}}(i, i)$ is the $i^{\text {th }}$ diagonal coefficient of $C_{\hat{\chi} \hat{x}}$

The relative standard deviation $\% \sigma_{\hat{\chi}_{r}}$ is given by:

$\% \sigma_{\hat{\chi}_{r i}}=100 \sigma_{\hat{\chi}_{i}}|| \hat{\chi}_{i} \mid$, for $\left|\hat{\chi}_{i}\right| \neq 0$

The ordinary LS can be improved by taking into account different standard deviations on joint $j$ equations errors [16]. Data in $Y$ and $W$ of (6) are sorted and weighted with 
the inverse of the standard deviation of the error calculated from OLS solution of the equations of joint $j$ [16].

This weighting operation normalises the errors in (6) and gives the weighted LS estimation of the parameters (IDIMWLS).

\section{Global IdENTIFICATION OF THE JoINT Drive Gains}

\section{A. IDIM Including a Payload and Drive Gains}

The payload is considered as a link $n+1$ fixed to the link $n$ of the robot. Only $n_{L}$ of its parameters are considered known. The model (4) becomes:

$$
\tau=v_{\tau} g_{\tau}=\left[\begin{array}{lll}
\Phi & \Phi_{u L} & \Phi_{k L}
\end{array}\right]\left[\begin{array}{lll}
\chi^{T} & \chi_{u L}^{T} & \chi_{k L}^{T}
\end{array}\right]^{T}+e
$$

where:

$\chi_{k L}$ is the $\left(n_{L} \times 1\right)$ vector of the known inertial parameters of the payload;

$\chi_{u L}$ is the $\left(\left(10-n_{L}\right) \times 1\right)$ vector of the unknown inertial parameters of the payload,

$\Phi_{k L}$ is the $\left(n \times n_{L}\right)$ jacobian matrix of $\tau_{i d m}$, with respect to the vector $\chi_{k L}$,

$\Phi_{u L}$ is the $\left(n \times\left(10-n_{L}\right)\right)$ jacobian matrix of $\tau_{i d m}$, with respect to the vector $\chi_{u L}$.

\section{B. Total Least Squares Identification of the Drive Gains (IDIM-TLS)}

Details on the TLS identification method can be found in [19] and many papers of the same authors. This method has been applied in [18] for the identification of the drive gains and the dynamic parameters on a two degrees of freedom robot (dof) but gives arguable results due to the lack of an accurate scale factor. In this paper we propose a major improvement with the scaling of parameters using the accurate value of an additional payload mass.

In order to identify the payload parameters, it is necessary that the robot carried out two trajectories: (a) without the payload and (b) with the payload fixed to the end-effector [17]. The sampling and filtering of the model IDIM (12) can be then written as:

$Y=\left[\begin{array}{c}V_{\tau a} \\ V_{\tau b}\end{array}\right] g_{\tau}=\left[\begin{array}{ccc}W_{a} & 0 & 0 \\ W_{b} & W_{u L} & W_{k L}\end{array}\right]\left[\begin{array}{lll}\chi^{T} & \chi_{u L}^{T} & \chi_{k L}^{T}\end{array}\right]^{T}+\rho$

where:

$V_{\tau a}$ is the matrix of $v_{\tau}$ samples in the unloaded case,

$V_{\tau b}$ is the matrix of $v_{\tau}$ samples in the loaded case,

$V_{\tau i}=\left[\begin{array}{cccc}V_{\tau i}^{1} & 0 & \cdots & 0 \\ 0 & V_{\tau i}^{2} & \cdots & 0 \\ \vdots & \vdots & \ddots & \vdots \\ 0 & 0 & \cdots & V_{\tau i}^{n}\end{array}\right], \quad V_{\tau i}^{j}=\left[\begin{array}{c}v_{\tau i, 1}^{j} \\ v_{\tau i, 2}^{j} \\ \vdots \\ v_{\tau i, r / n}^{j}\end{array}\right]$, with $i=a, b$

$v_{x i, k}^{j}$ is the $k$-th sample of current reference for actuator $j$,

$W_{a}$ is the observation matrix of the robot in the unloaded case,
$W_{b}$ is the observation matrix of the robot in the loaded case,

$W_{u L}$ is the observation matrix of the robot corresponding to the unknown payload inertial parameters,

$W_{k L}$ is the observation matrix of the robot corresponding to the known payload inertial parameters. Eq. (13) becomes: $W_{\text {tot }} \chi_{\text {tot }}=\rho$

where

$W_{t o t}=\left[\begin{array}{cccc}-W_{a} & V_{a} & 0 & 0 \\ -W_{b} & V_{b} & -W_{u L} & -W_{k L}\end{array}\right]$ is a $\left(r_{t o t} \times r_{t o t}\right)$ matrix,

and $\chi_{\text {tot }}=\left[\begin{array}{llll}\chi^{T} & g_{\tau}^{T} & \chi_{u L}^{T} & \chi_{k L}^{T}\end{array}\right]^{T}$ is a $\left(n_{b}+n+10\right)$ vector.

Without perturbation, $\rho=0$ and $W_{\text {tot }}$ should be rank deficient to get the solutions $\lambda \chi_{\text {tot }} \neq 0$ depending on a scale coefficient $\lambda$. However because of the measurement perturbations, $W_{\text {tot }}$ is a full rank matrix. Therefore, the system (15) is replaced by the compatible system closest to (15) with respect to the Frobenius norm:

$\hat{W}_{\text {tot }} \hat{\chi}_{\text {tot }}=0, \hat{\chi}_{\text {tot }}=\left[\hat{\chi}^{T}, \hat{g}_{\tau}^{T}, \hat{\chi}_{u L}^{T}, \hat{\chi}_{k L}^{T}\right]^{T}$

where $\hat{W}_{\text {tot }}$ is the rank deficient matrix, with the same dimension as $W_{t o t}$, which minimizes the Frobenius norm $\left\|W_{\text {tot }}-\hat{W}_{\text {tot }}\right\|_{F}$.

$\hat{\chi}_{\text {tot }}$ is the solution of the compatible system closest to (15). $\hat{W}_{\text {tot }}$ can be computed thanks to the Singular Value Decomposition (SVD) of $W_{\text {tot }}$ [20]:

$W_{\text {tot }}=U\left[\begin{array}{c}\operatorname{diag}\left(\sigma_{i}\right) \\ 0\end{array}\right] V^{T}$,

where $U$ and $V$ are $\left(r_{\text {tot }} \times r_{\text {tot }}\right)$ and $\left(\left(n_{b}+n+10\right) \times\left(n_{b}+n+10\right)\right)$ orthonormal matrices, respectively, and $\operatorname{diag}\left(\sigma_{i}\right)$ is a $\left(\left(n_{b}+n+10\right) \times\left(n_{b}+n+10\right)\right)$ diagonal matrix with singular values $\sigma_{i}$ of $W_{\text {tot }}$ sorted in decreasing order. The solution of (16) is given by:

$\hat{W}_{\text {tot }}=W_{t o t}-\sigma_{n+n_{b}} U_{n+n_{b}} V_{n+n_{b}}^{T}$,

where $\sigma_{n+n_{b}}$ is the smallest singular value of $W_{\text {tot }}$ and $U_{n+n_{b}}$ $\left(V_{n+n_{b}}\right.$, resp.) the column of $U$ ( $V$, resp.) corresponding to $\sigma_{n+n_{b}}$. Then, the normalized optimal solution $\hat{\chi}_{\text {tot }}^{l}$ $\left(\left\|\hat{\chi}_{\text {tot }}^{l}\right\|=1\right)$ is given by the last column of $V, \hat{\chi}_{\text {tot }}^{l}=V_{n+n_{b}}[18]$.

There are infinity of vectors $\hat{\chi}_{\text {tot }}=\lambda \hat{\chi}_{\text {tot }}^{l}$ that can be obtained by a scale factor $\lambda$. A unique solution $\hat{\chi}_{\text {tot }}^{*}=\hat{\lambda} \hat{\chi}_{\text {tot }}^{l}$ can be found by taking into account the known values $\chi_{k L}$ of the payload parameters and their corresponding identified values $\hat{\chi}_{k L}^{l}$. The optimal scale factor $\hat{\lambda}$ can be calculated as the LS solution of :

$\chi_{k L}=\hat{\chi}_{k L}^{l} \lambda+\rho_{k L}$ 


\section{Discussion on the A Priori Knowledge of the Payload Parameters}

The accuracy of $\hat{\lambda}$ depends on the accuracy of $\chi_{k L}$, depending on the knowledge of the payload parameters, and on the accuracy of $\hat{\chi}_{k L}^{l}$ in (19).

The most accurate payload parameter is the mass value $M_{L}$ that can be accurately measured using a weighing machine.

Then in the next section we shall compare 2 solutions:

$\hat{\lambda}_{1}=\hat{\chi}_{k L}^{l+} \chi_{k L}$

where $\hat{\lambda}_{l}$ is the LS solution of (19) calculated with all known payload parameters, and:

$$
\hat{\lambda}_{2}=M_{L} / \hat{M}_{L}^{l}
$$

where $\hat{M}_{L}^{l}$ is the identified value of $M_{L}$ in $\hat{\chi}_{\text {tot }}^{l}$.

It will be shown in the next section, that the experimental results confirm the efficiency of the approach that uses the mass only.

\section{CASe Study}

\section{A. Description of the TX 40 Kinematics}

The Stäubli TX-40 robot (Fig. 1) has a serial structure with six rotational joints. Its kinematics is defined using the modified Denavit and Hartenberg notation (MDH) [12]. In this notation, the link $j$ fixed frame is defined such that the $z_{j}$ axis is taken along joint $j$ axis and the $x_{j}$ axis is along the common normal between $z_{j}$ and $z_{j+1}$ (Fig. 1). The geometric parameters defining the robot frames are given in Table 1. The payload is denoted as the link 7. The parameter $\sigma_{j}=0$, means that joint $j$ is rotational, $\alpha_{j}$ and $d_{j}$ parameterize the angle and distance between $z_{j-l}$ and $z_{j}$ along $x_{j-1}$, respectively, whereas $\theta_{j}$ and $r_{j}$ parameterize the angle and distance between $x_{j-1}$ and $x_{j}$ along $z_{j}$, respectively. For link $7, \sigma_{j}=2$ means that the link 7 is fixed on the link 6 . Since all the joints are rotational then the $\mathrm{MDH}$ position $\theta_{j}$ is equal to the joint position $q_{j}$ given by the CS8C controller of the TX-40 robot, except for joints 2 and 3 where the $\mathrm{MDH}$ notation differs the Staübli variables, $\theta_{2}=q_{2}-\pi / 2, \theta_{3}=q_{3}+\pi / 2$.

The TX40 robot is characterized by a coupling between the joints 5 and 6 such that:

$$
\left[\begin{array}{l}
\dot{q} r_{5} \\
\dot{q} r_{6}
\end{array}\right]=\left[\begin{array}{cc}
K 5 & 0 \\
K 6 & K 6
\end{array}\right]\left[\begin{array}{l}
\dot{q}_{5} \\
\dot{q}_{6}
\end{array}\right],\left[\begin{array}{l}
\tau_{c_{5}} \\
\tau_{c_{6}}
\end{array}\right]=\left[\begin{array}{cc}
K 5 & K 6 \\
0 & K 6
\end{array}\right]\left[\begin{array}{l}
\tau_{r_{5}} \\
\tau_{r_{6}}
\end{array}\right]
$$

where $\dot{q} r_{j}$ is the velocity of the rotor of motor $j, \dot{q}_{j}$ is the velocity of joint $j, K 5$ is the transmission gain ratio of axis 5 and $K 6$ is the transmission gain ratio of axis $6, \tau_{c j}$ is the

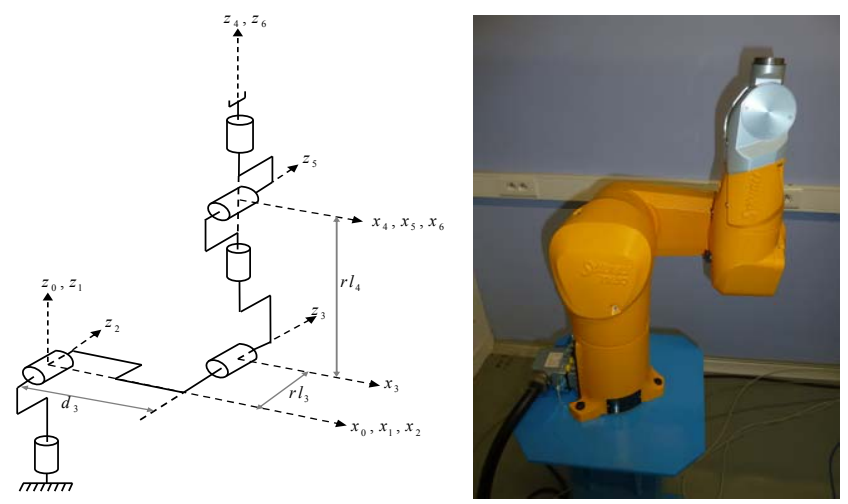

Fig. 1. Link frames of the TX-40 robot

\begin{tabular}{|c|c|c|c|c|c|}
\hline$j$ & $\sigma_{j}$ & $\alpha_{j}$ & $d_{j}$ & $\theta_{j}$ & $r_{j}$ \\
\hline 1 & 0 & 0 & 0 & $q_{1}$ & 0 \\
\hline 2 & 0 & $-\pi / 2$ & 0 & $q_{2}-\pi / 2$ & 0 \\
\hline 3 & 0 & 0 & $d_{3}=0.225(\mathrm{~m})$ & $q_{3}+\pi / 2$ & $r l_{3}=0.035(\mathrm{~m})$ \\
\hline 4 & 0 & $+\pi / 2$ & 0 & $q_{4}$ & $r l_{4}=0.225(\mathrm{~m})$ \\
\hline 5 & 0 & $-\pi / 2$ & 0 & $q_{5}$ & 0 \\
\hline 6 & 0 & $+\pi / 2$ & 0 & $q_{6}$ & 0 \\
\hline 7 & 2 & 0 & 0 & 0 & 0 \\
\hline
\end{tabular}

TABLE I

motor torque of joint $j$, taking into account the coupling effect, $\tau_{r j}$ is the electro-magnetic torque of the rotor of motor $j$. With the coupling between joints 5 and 6 , (5) and (14) becomes:

$\tau=v_{\tau} g_{\tau}=\left[\begin{array}{cccc}v_{\tau}^{1} & 0 & \cdots & 0 \\ 0 & \vdots & \cdots & 0 \\ \vdots & \vdots & v_{\tau}^{5} & v_{\tau}^{6} \\ 0 & 0 & 0 & v_{\tau}^{6}\end{array}\right]\left[\begin{array}{c}g_{\tau}^{1} \\ \vdots \\ g_{\tau}^{5} \\ g_{\tau}^{6}\end{array}\right]$,

and $V_{\tau i}=\left[\begin{array}{cccc}V_{\tau i}^{1} & 0 & \cdots & 0 \\ 0 & \vdots & \cdots & 0 \\ \vdots & \vdots & V_{\tau i}^{5} & V_{\tau i}^{6} \\ 0 & 0 & 0 & V_{\tau i}^{6}\end{array}\right]$

The coupling between joints 5 and 6 also adds the effect of the inertia of rotor 6 and new viscous and Coulomb friction parameters $f v_{m 6}$ and $f c_{m 6}$, to both $\tau_{c 5}$ and $\tau_{c 6}$.

We can write:

$\tau_{c_{5}}=\tau_{5}+I a_{6} \ddot{q}_{6}+f v m_{6} \dot{q}_{6}+f c m_{6} \operatorname{sign}\left(\dot{q}_{6}\right)$ and

$\tau_{c_{6}}=\tau_{6}+I a_{6} \ddot{q}_{5}+f v m_{6} \dot{q}_{5}+f c m_{6}\left(\operatorname{sign}\left(\dot{q}_{5}+\dot{q}_{6}\right)-\operatorname{sign}\left(\dot{q}_{6}\right)\right)$. where $\tau_{5}, \quad \tau_{6}$ already contain the terms $\left(\operatorname{Ia} \ddot{q}_{j}+f v_{j} \dot{q}_{j}+f c_{j} \operatorname{sign}\left(\dot{q}_{j}\right)\right)$, for $j=5$ and 6 respectively,

$I a_{5}=K_{5}^{2} J a_{5}+K_{6}^{2} J a_{6}$ and $I a_{6}=K_{6}^{2} J a_{6}$

$J a_{j}$ is the moment of inertia of rotor $j$.

The TX40 has $N_{s}=86$ standard dynamic parameters given by the $14 \times 6$ usual standard parameters, plus $f v_{m 6}$ and $f c_{m 6}$.

\section{B. Identification of the Drive Gains}

The proposed method is validated using a calibrated payload (Fig. 2). 


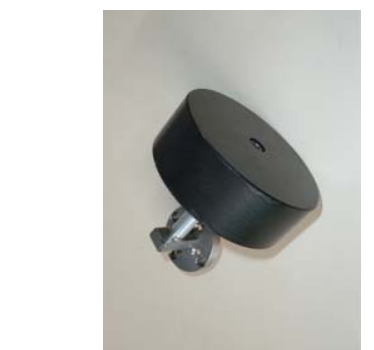

Fig. 2. The $4.59 \mathrm{Kg}$ payload

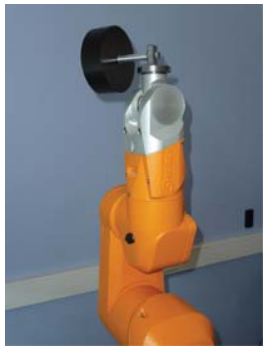

Its mass has been measured with a weighing machine $\left(M_{L}=4.59 \mathrm{Kg} \pm 0.05 \mathrm{Kg}\right)$. The other parameters have been calculated using CAD software. They are given in table 2. Their values are accurate due to the simplicity of the payload shape (Fig. 2).

Three different identifications of the payload inertia parameters are achieved:

- Case 1: the payload parameters are identified using the manufacturer's drive gains

- Case 2: the drive gains are first identified with the base parameters with IDIM-TLS using (20) calculated with all known payload parameters. They are then used in order to reidentifiy the payload parameters and the robot dynamic parameters with IDIM-WLS.

- Case 3: the drive gains are first identified with the base parameters with IDIM-TLS using (21) calculated with only the mass of the payload. They are then used in order to reidentifiy the payload parameters and the robot dynamic parameters with IDIM-WLS.

The a priori drive gains (Case 1) and the identified ones (Cases 2 and 3) are given in table 3. For each joints, the identified values are close for the manufacturer's values, but the mean error is about $11 \%$. The maximal error grows up to $21 \%$ !

The identified values of the payload inertial parameters are presented in table 2. Moreover, the quality of identification is detailed at table 4. It appears that the identified gains lead to the best results. Moreover, the best model identification is achieved with the use of the TLS with only the mass. This result is really appealing for industrial applications and show the efficiency and the
TABLE 3

IDENTIFIED DRIVE GAINS

Joint 1 Joint 2 Joint 3 Joint 4 Joint 5 Joint 6

\begin{tabular}{cccccccc}
\hline \hline \multicolumn{1}{c}{ A priori val. } & $g_{\tau}^{j}$ & 32.96 & 32.96 & 25.65 & -11.52 & 18.48 & 7.68 \\
Case 2 & $g_{\tau}^{j}$ & 35.22 & 32.02 & 23.92 & -9.05 & 15.49 & 6.55 \\
& $2 \sigma_{\tilde{x}_{i}}$ & 0.540 & 0.483 & 0.372 & 0.015 & 0.252 & 0.011 \\
& $\% \sigma_{\hat{\chi}_{i}}$ & 1.53 & 1.51 & 1.56 & 1.67 & 1.63 & 1.63 \\
Case 3 & $g_{\tau}^{j}$ & $\mathbf{3 5 . 2 4}$ & $\mathbf{3 2 . 0 4}$ & $\mathbf{2 3 . 9 4}$ & $\mathbf{- 9 . 0 5}$ & $\mathbf{1 5 . 5 0}$ & $\mathbf{6 . 5 6}$ \\
& $2 \sigma_{\hat{\chi}_{i}}$ & 0.540 & 0.484 & 0.372 & 0.015 & 0.252 & 0.011 \\
& $\% \sigma_{\hat{x}_{i}}$ & 1.53 & 1.51 & 1.56 & 1.67 & 1.63 & 1.64 \\
\hline \hline
\end{tabular}

TABLE 4

QUALITY OF IDENTIFICATION.

\begin{tabular}{lccc}
\hline \hline & Error norm $\|\hat{\rho}\|$ & Relative Error norm $\|\hat{\rho}\| /\|Y\|$ & $\hat{\sigma}_{\rho}$ \\
\hline Case 1 & 33.8366 & 0.043245 & 0.495164 \\
Case 2 & 31.6252 & 0.0403937 & 0.462829 \\
Case 3 & $\mathbf{3 1 . 4 7 8 3}$ & $\mathbf{0 . 0 4 0 2 8 4 6}$ & $\mathbf{0 . 4 6 0 6 8}$ \\
\hline \hline
\end{tabular}

$\|\hat{\rho}\|=\|Y-W \hat{\chi}\|$ is the minimal norm of error, $\hat{\sigma}_{\rho}$ is given by (9).

simplicity of the method.

Finally, in order to validate the new drive gain values a new payload is identified (Table 5). The parameters are very close to the a priori ones in all cases but the best identification is still obtained with the gains of Case 3. The torques calculated with the model (12) identified with the gains of Case 3 are presented in Fig. 3. It is possible to conclude that the drive gains have been well identified with the IDIM-TLS.

\section{CONCLUSION}

This paper has presented a new method for the global identification of the total drive gains for robot joints. This method is easy to implement and does not need any special test or measurement on elements inside the joint drive train. It is based on a IDIM-TLS technique using current reference and position sampled data while the robot is tracking one reference trajectory without load fixed on the robot and one trajectory with a known payload fixed on the robot, whose inertial parameters are measured or calculated by a CAD model. The method has been experimentally validated on an industrial Stäubli TX-40 robot. Using the identified drive gains, the identification of the total dynamic model has been improved and another payload has been accurately

TABLE 2

IDENTIFICATION OF THE PAYLOAD DYNAMIC PARAMETERS.

\begin{tabular}{|c|c|c|c|c|c|c|c|c|c|c|}
\hline \multirow[b]{2}{*}{ Parameter } & \multirow[b]{2}{*}{ A priori value } & \multicolumn{3}{|c|}{ Case 1} & \multicolumn{2}{|c|}{ Case 2} & \multirow[b]{2}{*}{$\% \sigma_{\hat{\chi}_{n}}$} & \multicolumn{3}{|c|}{ Case 3} \\
\hline & & Identified values & $2 \sigma_{\hat{\chi}_{i}}$ & $\% \sigma_{\hat{\chi}_{i}}$ & Identified values & $2 \sigma_{\hat{\chi}_{i}}$ & & Identified values & $2 \sigma_{\hat{\chi}_{i}}$ & $\% \sigma_{\hat{\chi}_{n i}}$ \\
\hline$X X_{L}$ & $0.64 e-1$ & $1.12 \mathrm{e}-1$ & $1.99 \mathrm{e}-3$ & 1.8 & $9.02 \mathrm{e}-2$ & $1.54 \mathrm{e}-3$ & 1.7 & $9.24 \mathrm{e}-2$ & $1.45 \mathrm{e}-3$ & 1.6 \\
\hline$X Y_{L}$ & $-1.80 e-2$ & $-1.83 e-2$ & $7.21 \mathrm{e}-4$ & 3.9 & $-1.34 \mathrm{e}-2$ & $6.65 \mathrm{e}-4$ & 5.0 & $-1.45 e-2$ & $6.75 e-4$ & 4.7 \\
\hline$X Z_{L}$ & $2.60 e-2$ & $2.93 \mathrm{e}-2$ & $8.88 \mathrm{e}-4$ & 3.0 & $1.96 \mathrm{e}-2$ & $5.91 \mathrm{e}-4$ & 3.0 & $1.93 \mathrm{e}-2$ & $5.90 \mathrm{e}-4$ & 3.1 \\
\hline$Y Y_{L}$ & $0.64 e-1$ & $1.15 \mathrm{e}-1$ & $1.80 \mathrm{e}-3$ & 1.6 & $9.12 \mathrm{e}-2$ & $1.41 \mathrm{e}-3$ & 1.5 & $8.77 \mathrm{e}-2$ & $1.44 \mathrm{e}-3$ & 1.6 \\
\hline$Y Z_{L}$ & $2.60 e-2$ & $4.15 \mathrm{e}-2$ & $6.32 \mathrm{e}-4$ & 1.5 & $3.59 \mathrm{e}-2$ & $4.93 \mathrm{e}-4$ & 1.4 & $3.75 \mathrm{e}-2$ & $6.45 e-4$ & 1.7 \\
\hline$Z Z_{L}$ & $4.40 e-2$ & $7.02 \mathrm{e}-2$ & $5.78 \mathrm{e}-4$ & 0.8 & $6.00 \mathrm{e}-2$ & $4.68 \mathrm{e}-4$ & 0.8 & $6.01 \mathrm{e}-2$ & $4.69 \mathrm{e}-4$ & 0.8 \\
\hline$M X_{L}$ & $-2.90 e-1$ & $-3.02 \mathrm{e}-1$ & $2.47 \mathrm{e}-3$ & 0.8 & $-2.82 \mathrm{e}-1$ & $2.35 \mathrm{e}-3$ & 0.8 & $-2.83 e-1$ & $2.35 \mathrm{e}-3$ & 0.8 \\
\hline$M Y_{L}$ & $-2.90 e-1$ & $-3.20 \mathrm{e}-1$ & $2.55 \mathrm{e}-3$ & 0.8 & $-2.82 \mathrm{e}-1$ & $1.84 \mathrm{e}-3$ & 0.7 & $-2.81 \mathrm{e}-1$ & $1.83 \mathrm{e}-3$ & 0.7 \\
\hline$M Z_{L}$ & $4.10 e-1$ & $5.18 \mathrm{e}-1$ & $4.38 \mathrm{e}-3$ & 0.8 & $4.42 \mathrm{e}-1$ & $3.41 \mathrm{e}-3$ & 0.8 & $4.44 \mathrm{e}-1$ & $3.39 \mathrm{e}-3$ & 0.8 \\
\hline$M_{L}$ & 4.59 & 4.48 & $3.02 \mathrm{e}-2$ & 0.7 & 4.58 & $2.73 \mathrm{e}-2$ & 0.6 & 4.58 & $2.72 \mathrm{e}-2$ & 0.6 \\
\hline
\end{tabular}



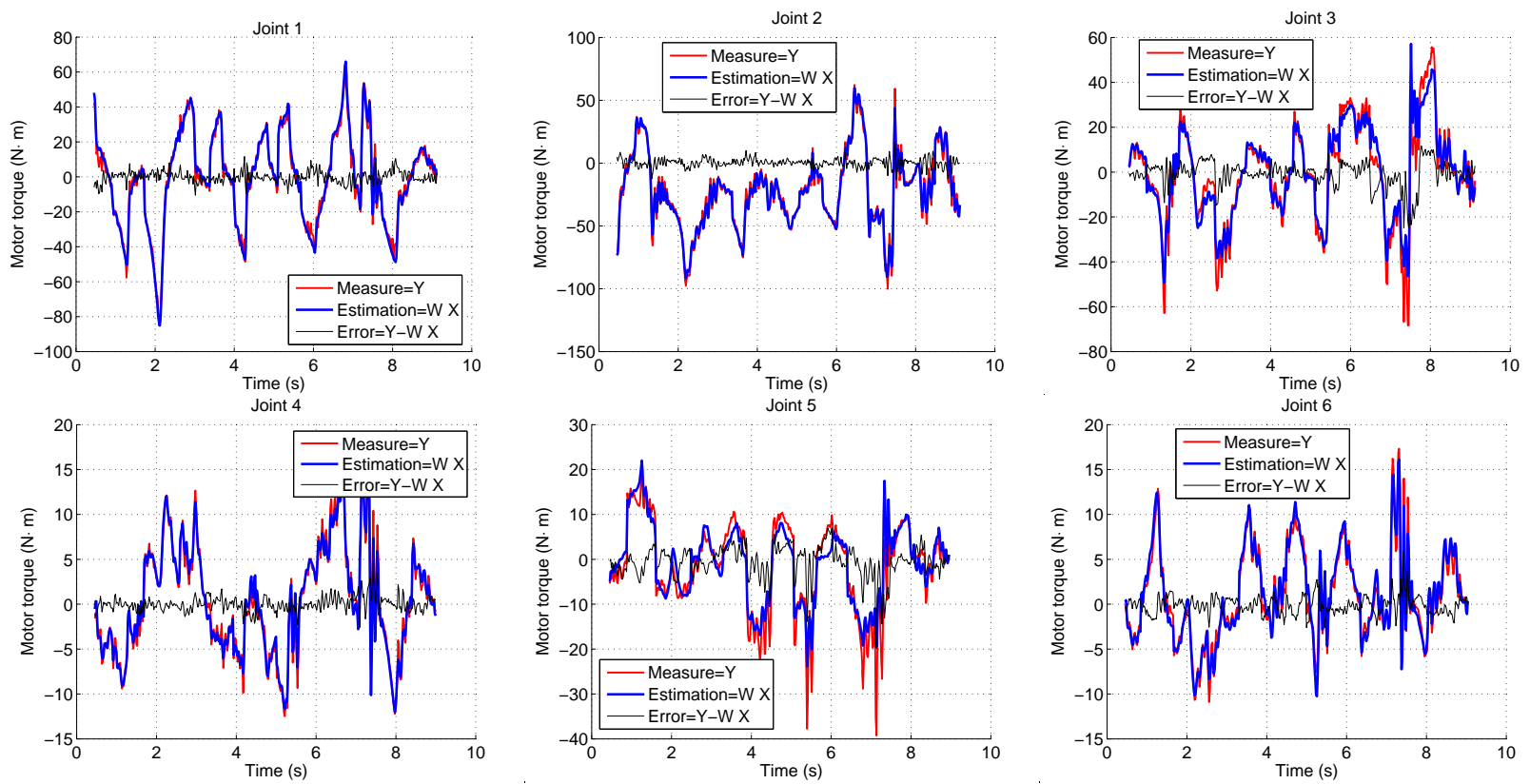

Fig. 3. Measured and reconstructed torques of the TX-40

TABLE 5

IDENTIFICATION OF THE NEW PAYLOAD DYNAMIC PARAMETERS.

\begin{tabular}{|c|c|c|c|c|c|c|c|c|c|c|}
\hline \multirow[b]{2}{*}{ Parameter } & \multirow[b]{2}{*}{ A priori value } & \multicolumn{3}{|c|}{ Case 1} & \multicolumn{3}{|c|}{ Case 2} & \multicolumn{3}{|c|}{ Case 3} \\
\hline & & Identified values & $2 \sigma_{\hat{\chi}_{i}}$ & $\% \sigma_{\hat{z}_{n}}$ & Identified values & $2 \sigma_{\hat{z}_{i}}$ & $\% \sigma_{\hat{\chi}_{n}}$ & Identified values & $2 \sigma_{\hat{\chi}_{i}}$ & $\% \sigma_{\hat{x}_{n}}$ \\
\hline$X X_{L}$ & $1.51 e-2$ & $1.57 \mathrm{e}-2$ & $8.75 \mathrm{e}-4$ & 5.6 & $1.35 \mathrm{e}-2$ & $7.64 \mathrm{e}-4$ & 5.7 & $1.15 \mathrm{e}-2$ & $8.29 \mathrm{e}-4$ & 7.2 \\
\hline$X Y_{L}$ & $-9.06 e-4$ & $-2.41 \mathrm{e}-3$ & $3.16 \mathrm{e}-4$ & 13.1 & $-1.60 \mathrm{e}-3$ & $3.47 \mathrm{e}-4$ & 21.7 & $-1.71 \mathrm{e}-3$ & $3.52 \mathrm{e}-4$ & 20.6 \\
\hline$X Z_{L}$ & $3.61 e-3$ & $4.21 \mathrm{e}-3$ & $3.34 \mathrm{e}-4$ & 7.9 & $4.04 \mathrm{e}-3$ & $2.91 \mathrm{e}-4$ & 7.2 & $3.95 e-3$ & $3.84 \mathrm{e}-4$ & 9.7 \\
\hline$Y Y_{L}$ & $1.51 e-2$ & $1.40 \mathrm{e}-2$ & $8.95 \mathrm{e}-4$ & 6.4 & $1.24 \mathrm{e}-2$ & $7.78 \mathrm{e}-4$ & 6.3 & $1.06 e-2$ & $7.71 \mathrm{e}-4$ & 7.3 \\
\hline$Y Z_{L}$ & $3.61 e-3$ & $1.93 \mathrm{e}-3$ & $3.00 \mathrm{e}-4$ & 15.5 & $1.05 \mathrm{e}-3$ & $2.63 \mathrm{e}-4$ & 25.1 & $1.59 \mathrm{e}-3$ & $2.63 \mathrm{e}-4$ & 16.5 \\
\hline$Z Z_{L}$ & $3.44 e-3$ & $3.88 \mathrm{e}-3$ & $2.88 \mathrm{e}-4$ & 7.4 & $1.26 \mathrm{e}-3$ & $2.85 \mathrm{e}-4$ & 22.7 & $3.26 e-3$ & $2.55 \mathrm{e}-4$ & 7.8 \\
\hline$M X_{L}$ & $-4.00 e-2$ & $-2.37 \mathrm{e}-2$ & $1.59 \mathrm{e}-3$ & 4.7 & $-2.58 \mathrm{e}-2$ & $1.29 \mathrm{e}-3$ & 5.0 & $-2.73 e-2$ & $1.31 \mathrm{e}-3$ & 4.8 \\
\hline$M Y_{L}$ & $-3.99 e-2$ & $-4.18 \mathrm{e}-2$ & $1.41 \mathrm{e}-3$ & 3.4 & $-3.52 \mathrm{e}-2$ & $1.17 \mathrm{e}-3$ & 3.3 & $-3.49 \mathrm{e}-2$ & $1.18 \mathrm{e}-3$ & 3.4 \\
\hline$M Z_{L}$ & 0.15 & 0.192 & $2.51 \mathrm{e}-3$ & 1.3 & 0.161 & $2.24 \mathrm{e}-3$ & 1.4 & 0.161 & $2.26 \mathrm{e}-3$ & 1.4 \\
\hline$M_{L}$ & 1.686 & 1.66 & $1.74 \mathrm{e}-2$ & 1.0 & 1.67 & $1.74 \mathrm{e}-2$ & 1.0 & 1.68 & $1.76 \mathrm{e}-2$ & 1.0 \\
\hline
\end{tabular}

$\sigma_{\hat{\chi}_{i}}$ is the standard deviation and $\% \sigma_{\hat{\chi}_{n}}$ its relative value

identified. This shows the effectiveness of the method.

\section{REFERENCES}

[1] C. Canudas de Wit and A. Aubin, "Parameters identification of robots manipulators via sequential hybrid estimation algorithms", Proc. IFAC Congress, Tallin, 1990, pp. 178-183.

[2] M. Gautier, "Identification of robots dynamics", Proc. IFAC Symp. on Theory of Robots, Vienne, Austria, December 1986, p. 351-356.

[3] M. Gautier and W. Khalil, "Direct calculation of minimum set of inertial parameters of serial robots", IEEE Trans. on Robotics and Automation, Vol. RA-6(3), 1990, p. 368-373.

[4] J. Hollerbach, W. Khalil, M. Gautier, "Model Identification", chapter 14 in Siciliano Bruno, and Oussama Khatib. eds « Springer Handbook of Robotics », Springer, 2008.

[5] W. Khalil and E. Dombre, "Modeling, identification and control of robots", Hermes Penton London, 2002.

[6] P.K. Khosla and T. Kanade, "Parameter identification of robot dynamics", Proc. $24^{\text {th }}$ IEEE CDC, Fort-Lauderdale, 1985, p. 17541760.

[7] Z. Lu, K.B. Shimoga and A. Goldenberg, "Experimental determination of dynamic parameters of robotic arms", Journal of Robotics Systems, Vol. 10, N8, 1993, p.1009-1029.

[8] P.P. Restrepo and M. Gautier, "Calibration of drive chain of robot joints," Proceedings of the 4th IEEE Conference on Control Applications, 1995, pp. 526-531.

[9] P. Corke, "In situ measurement of robot motor electrical constants," Robotica, vol. 23, no. 14, pp.433-436, 1996.
[10] M. Gautier and S. Briot, "New Method for Global Identification of the Joint Drive Gains of Robots using a Known Inertial Payload", Proc. IEEE ECC CDC, December 12-15, 2011, Orlando, Florida, USA.

[11] M. Gautier and S. Briot, "New Method for Global Identification of the Joint Drive Gains of Robots using a Known Payload Mass", Proc. IEEE IROS., Sept., 2011, San Fran., USA.

[12] W. Khalil and J.F. Kleinfinger, "A new geometric notation for open and closed loop robots", Proc. IEEE ICRA, 1986, San Francisco.

[13] R. Featherstone, D.E. Orin, "Dynamics", chapter 2 in Siciliano Bruno, and Oussama Khatib. eds "Springer Handbook of Robotics", Springer, 2008.

[14] M. Gautier, "Numerical calculation of the base inertial parameters", Journal of Robotics Systems, Vol. 8, ํ4, 1991, pp. 485-506.

[15] H. Mayeda, K. Yoshida and K. Osuka, "Base parameters of manipulator dynamic models", IEEE Trans. on Robotics and Automation, Vol. RA-6(3), 1990, p. 312-321.

[16] M. Gautier, "Dynamic identification of robots with power model", Proc. IEEE ICRA, 1997, Albuquerque, USA, April, pp. 1922-1927.

[17] W. Khalil, M. Gautier and P. Lemoine, "Identification of the payload inertial parameters of industrial manipulators," Proc. IEEE ICRA, 2007, Roma, Italy, April 10-14, pp. 4943-4948

[18] M Gautier, P. Vandanjon and C. Presse, "Identification of inertial and drive gain parameters of robots," Proc. IEEE CDC, 1994, Lake Buena Vista, FL, USA, pp. 3764-3769.

[19] S. Van Huffel, J. Vandewalle, "The Total Least Squares Problem: Computational Aspects and Analysis." Frontiers in Applied Mathematics series, 9. Philadelphia, Pennsylvania: SIAM, 1991.

[20] G.H. Golub, C.F. Van Loan, "Matrix computation" J. Hopkins $2^{\text {nd }}$ Ed. 